\title{
Lactococcus lactis alleviates oxidative stress and colitis in mice
}

Administration of Lactococcus lactis was found to exert a beneficial effect on colitis in mice by lowering colonic epithelial oxidative stress via lysozyme-mediated release of the bacterial enzyme superoxide dismutase (SodA).

Inflammation is typically associated with the production of reactive oxygen species. In particular, chronic oxidative stress, as seen in IBD, leads to tissue destruction and disrupts the microbial balance. Some beneficial bacterial strains can help to prevent the damage. Previous work by Wendy Garrett's laboratory established that



Image produced in consultation with Jason H. Kim. fermented milk containing five bacterial strains could be used to reduce colitis in mice. This present study confirms that one of these five strains, L. lactis I-1631, which is not widely recognized as a beneficial microbe and is commonly used in dairy production, initiates a marked reduction in colonic inflammation in three different mouse models of colitis. The effect is not mediated by anti-inflammatory properties of the bacterium, but rather endogenous production of SodA, which detoxifies superoxide anions. In mice with colitis that were treated with $L$. lactis I-1631 in fermented milk, the levels of colonic epithelial superoxide radicals were lower than in untreated animals. In vitro assays using primary colonic epithelial cells with chemically stimulated radical production confirmed the antioxidative properties. Genetic deletion of sodA in L. lactis abrogated the positive effect on colitis.

As SodA is located in the cytoplasm of L. lactis and not secreted, the investigators were faced with the dilemma of how it could mediate an effect in the intestine of its host. "L. lactis needs to be cracked open by a host enzyme, lysozyme, to deliver SodA to an inflamed colon. Parts of the colon produce high levels of lysozyme that lyses or opens L. lactis so it can deliver SodA," explains Garrett.

Lysozyme expression was found to be substantially increased in the inflamed colon of mice and humans - a natural targeting mechanism to ensure L. lactis will only exert its effect where needed if used in therapy. Evidence suggests that SodA delivery via L. lactis would be more efficient than SodA administration alone because of the enzyme's short half-life.

"We would be thrilled to see our results applied to help patients with IBD," says Garrett.

Christine Weber

Original article Ballal, S. A. et al. Host lysozyme-mediated lysis of Lactococcus lactis facilitates delivery of colitisattenuating superoxide dismutase to inflamed colons. Proc. Natl Acad. Sci. USA doi:10.1073/pnas.1501897112 